investigate trends in ICS prescribing across the London Clinical Commissioning Groups (CCGs) and reasons for variation.

Methods The NHS London Procurement Partnership monitors prescribing across London. It has designed a dashboard of primary care prescribing initiatives in collaboration with London CCGs, one of which attempts to support optimised ICS prescribing. The prescribing data for the financial year 2014/15 was compared with 2013/14 for the 32 London CCGs and London as a whole.

Results All but 2 London CCGs increased their total ICS spend between 2014/15 and 2013/14. Altogether, this was an increase of $3 \%$ to $£ 67.6$ million. In $2014 / 15$, HDICS accounted for $20 \%$ of all ICS items prescribed (range 11-27\%), or $£ 23.5$ million (36\%) of total ICS spend (range 23-50\%). 7/32 CCGs reduced expenditure on HDICS by $>1 \%$ compared with 2013/14, with one achieving a $16 \%$ reduction. Reduction in HDICS prescribing occurred mostly in CCGs with respiratory integrated care teams.

Conclusion In light of the National Review of Asthma Deaths, the increase in total spend on ICS may be appropriate. However, $20 \%$ of all ICS prescribed in London are for HDICS preparations accounting for $36 \%$ of the total spend on ICS. With more than two fold variation across CCGs, some HDICS prescribing may be inappropriate and reduction may be associated with the presence of integrated care teams. If all CCGs in London could manage a $10 \%$ reduction in inappropriate HDICS prescribing, this could reduce the risk of side effects to patients and save over $£ 2$ million per annum.

\section{M20 A FIVE-YEAR ANALYSIS OF AN INTEGRATED COPD SERVICE IN HACKNEY, LONDON - IS THIS THE RIGHT DIRECTION?}

${ }^{1} \mathrm{~A}$ Garner, ${ }^{2} \mathrm{M}$ Hodson, ${ }^{3} \mathrm{G}$ Ketsetzis, ${ }^{2} \mathrm{~A}$ Bhowmik. ${ }^{1}$ City and Hackney Clinical Commissioning Group, London, UK; ${ }^{2} A C E R S$, Respiratory Department, Homerton University Hospital, London, UK; ${ }^{3}$ North East London Commissioning Support Unit, London, UK

\subsection{6/thoraxjnl-2015-207770.447}

In response to high mortality rates, high numbers of COPD admissions, poor quality of care and a lack of integration of services for people with COPD, City and Hackney Primary Care Trust tendered the provision of an acute- and community-based COPD service from Homerton University Hospital in 2009 (the Acute COPD Early Response Service: ACERS).

We studied the impact of ACERS on outcomes for COPD patients in City and Hackney including patient CAT scores, healthcare usage (admissions, length of stay and bed days) and place of death, from 2010 to 2015.

We found a decrease in COPD admissions (from 1.38 admissions per 1000 population to 1.24 per 1000 population) following the establishment of the ACERS service (compared to an increase seen nationally over this time) and a significant reduction in the number of bed days for COPD patients - from 1,817 per year to 1,200 per year (Mann Whitney U test Z-Score $3.6607, \mathrm{p}<0.05)$. This was alongside a significant increase in patients staying less than 2 days in hospital - from $27 \%$ to $34 \%$ (significant at $\mathrm{p}<0.0001$ ) reflecting the effect of the service on early discharge. We also found a significant increase in the number of patients dying outside of hospital (a proxy for quality of end of life care as most patients express wishes to be cared for and die in their own home or a hospice). The percentage of City and Hackney COPD patients dying outside of hospital increased from $24 \%$ to $42 \%$ following introduction of ACERS $(\mathrm{p}=0.00015)$. Patient satisfaction with the service was high and patients saw a clinically significant improvement in CAT scores (from 24 to $20, \mathrm{n}=69$ ) following intervention by the ACERS team.

This data was used in a locally developed economic model to determine the economic benefits of the ACERS team and whether therefore, when comparing to the cost of the service, this service was cost-saving overall. The model found that the impact on place of death and healthcare usage meant that ACERS had net monetary benefits to commissioners.

We conclude that an exemplar integrated COPD service can provide financial and other benefits to commissioners which equate to a cost saving service with high return on initial investment. ACERS has been expanded from its original remit to now include management of patients with asthma and bronchiectasis.

\section{M21 COMPARISON OF THE EFFECT OF A VENTILATION MULTIDISCIPLINARY MEETING ON UTILISATION OF CRITICAL CARE RESOURCES}

${ }^{1} \mathrm{~A}$ Bishopp, ${ }^{1} \mathrm{~N}$ Santana-Vaz, ${ }^{1} \mathrm{~B}$ Beauchamp, ${ }^{2} \mathrm{~B}$ Chakraborty, ${ }^{1} \mathrm{G}$ Raghuraman, ${ }^{1}$ R Mukherjee. 'Birmingham Heartlands Hospital, Birmingham, UK; ${ }^{2}$ School of Mathematics, University of Birmingham, Birmingham, UK

\subsection{6/thoraxjnl-2015-207770.448}

Introduction and objectives Optimal utilisation of critical care resources requires timely discharge of patients from critical care to appropriate wards. This represents a challenging and high risk transition. Local audits revealed that a few multimorbid patients with difficult respiratory weans accounted for $30 \%$ of critical care bed days. A weekly ventilation multidisciplinary team (VMDT) meeting combining respiratory and critical care expertise was established at a 692-bed hospital to improve management and resource use for this patient group. The effect was compared to a $2^{\text {nd }}$ hospital within the same trust without VMDT.

Method A retrospective comparison of 6 month periods before (period 1: 1/10/07-31/3/08) and after (period 2: 1/10/12-31/3/ 13) introducing VMDT was carried out using data collected for Intensive Care National Audit and Research Centre. The same data was collected for a sister hospital, belonging to the same trust, without VMDT. The numbers of discharges to a respiratory ward with non- invasive ventilation (NIV) facilities were compared with Chi-Square test. The numbers of level 1 critical care bed days were compared with $\mathrm{T}$ test.

Results In period 1, hospital 1 discharged 458 patients from critical care and hospital 2 discharged 456. In period 2 these figures were $494(\mathrm{p}=0.30)$ and $495(\mathrm{p}=0.84)$ respectively. There was no change to background parameters. The number of discharges to respiratory ward with NIV facilities increased significantly in hospital 1 (36 to $65, \mathrm{p}=0,011$ ) after VMDT. Whilst the number of patients discharged to respiratory ward increased in hospital 2 this was not significant ( 9 to $19, \mathrm{p}=0.13$ ). The number of level 1 bed days fell significantly (208 to $18, \mathrm{p}<$ 0.0000000001 ) in hospital 1. Hospital 2 saw an increase in level 1 days over the same period.

Conclusion Introduction of VMDT increased the proportion of respiratory patients discharged to a respiratory ward from critical care and reduced level one bed days in hospital 1 by expediting the discharge of complex respiratory wean patients thereby increasing patient flow and liberating critical care resources. The same reduction was not observed in the hospital 2 suggesting this effect was not due to trust wide changes in critical care practice. 